\title{
Sonographic Comparison of Peak Systolic Velocities of Fetal Middle Cerebral Artery among Normotensive and Hypertensive Mothers
}

\author{
Javeria Khan $^{1 *}$, Sidra Shanawer ${ }^{1}$, Memoona Siddique ${ }^{1}$, Aisha Altaf ${ }^{1}$, Dr. Raham Bacha ${ }^{2}$, Syed Muhammad Yousuf \\ Farooq $^{3}$ \\ ${ }^{1}$ MID, University Institute of Radiological Sciences and Medical Imaging Technologies (UIRSMIT), Faculty of Allied Health Sciences (FAHS), The \\ University of Lahore, 1- Km Raiwind Rd, Sultan Town, Lahore, Punjab, Pakistan \\ ${ }^{2} \mathrm{Ph} . \mathrm{D}$ (Ultrasound), University Institute of Radiological Sciences and Medical Imaging Technologies (UIRSMIT), Faculty of Allied Health Sciences \\ (FAHS), The University of Lahore, 1- Km Raiwind Rd, Sultan Town, Lahore, Punjab, Pakistan \\ ${ }^{3}$ Biostatistics, University Institute of Radiological Sciences and Medical Imaging Technologies (UIRSMIT), Faculty of Allied Health Sciences (FAHS), \\ The University of Lahore, 1- Km Raiwind Rd, Sultan Town, Lahore, Punjab, Pakistan
}

DOI: $10.36348 / \mathrm{sb} .2020 . v 06 i 04.002 \quad$ | Received: 03.01 .2020 | Accepted: 10.01 .2020 | Published: 30.04 .2020

*Corresponding author: Javeria Khan

\section{Abstract}

Background: Pregnancy induced hypertension is most common hypertension in expectant women after 20 weeks growing the risk of subsequent hypertension, ischemic heart disease and cerebrovascular disease. Reduced renovation of the spiral artery is particularly being well-thought-out as a cause failing. Maternal hypertension effects placental insufficiency, which can disturb the nutrition and the oxygen supply to the fetus. In hypertensive mothers, the potential of placental insufficiency is raised leading to intrauterine growth retardation but peak systolic velocity of middle cerebral artery has no reference value for our population. No matter, Ultrasonography can examine dynamically in real-time. However, the sonographic criteria in practice have no clear-cut numerical value to label IUGR. Objective: To compare peak systolic velocity of fetal middle cerebral artery among normotensive and hypertensive mothers. Setting: The data was collected from the following setups of Pakistan. 1) Mushtaq Medical Imaging Sargodha. 2) PAF Hospital Lahore. Materials and methods: The study was conducted after the approval of ethical committee of the University of Lahore. All patients were enrolled in this study after signing the informed consent form. Toshiba Xario with convex transducer of frequency 3-5 MHz was used. Fetal Middle cerebral artery indices were measured by Pulsed and Power Doppler through trans-abdominal scan. A cross sectional analytical sampling technique was used and data was further analyzed with the help of the Statistical Package for the Social Sciences version twenty-four (SPSS 24, International Business Machines, Armonk, NY, the United States of America). Results: Among 137 patients, 60(43.8\%) patients were hypertensive with mean PSV of the MCA, $35.63 \mathrm{~cm} / \mathrm{s}$ and $77(56.2 \%)$ normotensive patients with mean PSV of $36.64 \mathrm{~cm} / \mathrm{s}$. Conclusions: Peak systolic velocity of fetal middle cerebral artery in hypertensive mothers is less than that of Normotensive mothers.

Keywords: Ultrasound, Pregnancy, Hypertension, Placenta, peak systolic velocity.

Copyright @ 2020: This is an open-access article distributed under the terms of the Creative Commons Attribution license which permits unrestricted use, distribution, and reproduction in any medium for non-commercial use (NonCommercial, or CC-BY-NC) provided the original author and source are credited.

\section{INTRODUCTION}

When the systolic blood pressure is higher than $140 \mathrm{~mm}$ of $\mathrm{Hg}$ and diastolic blood pressure gives measurements greater than $90 \mathrm{~mm}$ of $\mathrm{Hg}$, this is known as hypertension [1]. Placenta is important for upholding of fetus during pregnancy and for helping usual growth and progress of fetus. Pregnancy-induced hypertension (PIH) or hypertension during pregnancy is the foremost reason of motherly transience and is a significant aspect in depletion of fetus [2]. Hypertension due to pregnancy enhances the threat of subsequent hypertension, ischemic heart disease and cerebrovascular disease [3].
The risk of pregnancy-induced hypertension is highest in women younger than 20 years; first conception; those with twin or molar pregnancies; those with hypercholesterolemia, pre gestational diabetes, or obesity; and those with a previous family history of hypertension due to pregnancy [4]. Placental ischemia/hypoxia is supposed to stimulate the initiation of the motherly vascular endothelium, which causes increase in vascular sensitivity to hormone angiotensin II and decrease in the production of vasodilators for example nitric oxide and these endothelial oddities cause generalized vasoconstriction in whole of the body 
including the kidneys, which have a dire participation in the longstanding maintenance of arterial pressure [5]. A placenta, but not the fetus, is requisite for the progress of hypertension during pregnancy. Certainly, the only active cure for hypertension during pregnancy is placental delivery. The old concept of the pathogenic processes convoluted in hypertension during pregnancy is that a placenta that is effected by ischemia yields soluble reasons that, when circulate through the mother's blood circulation, are accountable for the medical indices of the disease [6]. The source of hypertension thru pregnancy is still not known. Reduced remodeling of the spiral artery has particularly been measured as a flaw producing hypertension during pregnancy. Remodeling is a multistep course in which the first decidua associated step should be begun implantation may be occurring [7]. Usually, instantaneously after embedding, ectodermal cells form the outermost epithelial layer of the blastocyst increase to miscellaneous types of trophoblastic cell. Union of cells produces the syncytium below the embryo that is been implanted, which shows that the initial intrusive trophoblastic cell type migrating into the endometrium of the mother. When the space between the villi is formed, the inner layer of the trophoblasts originating from the trophectodermal layer forms primary villi by production and penetration through the primitive syncytium. All the way through pregnancy, these chief villi make subordinate and tertiary villi [8].

It is testified that the possibilities for disruption of placenta comprise of late age of mother at pregnancy and increased number of children, smoking, multiple gestations, chronic hypertension, pregnancy induced hypertension and oligohydramnios [9]. Because fetal growth anomalies are often supposed of as expressions of dysfunction of the placenta, it is remarkable to notice that placental abruption following pain, bleeding, and, possibly, interruption of fetal gas and nutrient exchange is more corporate in women with chronic hypertension [10]. Intrauterine growth restriction (IUGR) is frequently triggered by placental insufficiency, in reaction, fetus adjusts its own blood circulation to retain its nutrition and supply of oxygen to the brain ('brain-sparing') [11]. The Doppler detectable modifications in the fetal circulation related with IUGR and fetal hypoxia include raised resistance in the umbilical artery and peripheral vessel in connotation with reduced resistance in fetal cerebral vessels. This pattern of haemo dynamic adjustment is thought to mirror the "brain sparing" phenomenon in which hypoxic fetuses perfuse the brain, heart and adrenals [12].

As mentioned before, maternal hypertension can effect placental insufficiency, which in turn can disturb the nutrition and supply of oxygen to the fetus. It is pondered that to stun this insufficiency, fetal vascular remodeling takes place. During this vascular remodeling, most of the blood flow circulates to the vital organs. As middle cerebral artery supplies brain (vital organ), the middle cerebral artery will be targeted in the research to observe the effect of maternal blood pressure on fetal blood flow to brain. The supply of blood to the fetal brain can figure the growth of fetal brain. Middle cerebral artery peak systolic velocity may be a better analyst of perinatal impermanence in preterm IUGR than the PI. When the fetus is oxygen deficient, central reorganization of blood circulation outcomes in higher flow of blood to the fetal brain, heart, and adrenal glands, and a reduced flow of blood to the circulations to other tissues and organs of the fetus [13].

\section{METHODOLOGY}

In this cross-sectional study, data was collected from expectant women of age 20-35 years visiting mentioned diagnostic setups of Lahore and Sargodha during 4 months, from $10^{\text {th }}$ July to $10^{\text {th }}$ October 2019. Sample size of 137 patients was calculated by using standardized formula. The study was started after the approval of ethical committee of the University of Lahore. All the patients were enrolled in this study after signing the informed consent form. Data was collected according to the variable of the age, hypertension, parity, trimesters and presence or absence of IUGR. Ultrasound machine Toshiba Xario with convex transducer of frequency range 3 to $5 \mathrm{MHz}$ was used at Mushtaq Medical Imaging Sargodha and PAF Hospital Lahore, to calculate peak systolic velocities of fetal middle cerebral arteries trans abdominally. All the patients were examined according the American Institute of Ultrasound in Medicine (AIUM) gynecological ultrasound guidelines. Privacy of the patient was observed all the time and remained of top priority throughout the study.

Inclusion Criteria: Hypertensive and non-hypertensive mothers, pregnancy of $2^{\text {nd }}$ and $3^{\text {rd }}$ trimester, age group 20-35 years, nulliparous and multiparous mothers were included in the study.

Exclusion Criteria: Fetus with vital congenital abnormalities, intrauterine death of the babies and anomalies of the chromosomes were not included in the research study.

\section{Data Analysis}

The collected data was processed thorough Statistical Package for the Social Sciences version 24 (SPSS 24, IBM, Armonk, NY, United States of America) for statistical calculations and analysis. Frequency distribution and descriptive statistics of all involved variables and the incidence were calculated. 


\section{RESULTS}

In this study total frequency of the patients were 137 pregnant females with Pregnancy of $2^{\text {nd }}$ and $3^{\text {rd }}$ trimester. Individuals were of age group 20-35 years. Mean age of the patients was 29.81 2.81 (25-35years). Table number 1 shows descriptive statistics of normal and hypertensive patients. In total of 137 pregnant patients. Figure 3 and 4 show peak systolic velocities of Middle cerebral artery.
We diagnosed $60(43.8 \%)$ patients were hypertensive and $77(56.2 \%)$ were normotensive. Figure-1 and table no 1 show the frequency of hypertensive and normotensive patients. Out of 137 females 60(43.8\%) patients were diagnosed with hypertension and $77(56.2 \%)$ patients were normal (normotensive). Demographic details of Peak systolic velocity of middle cerebral artery are shown in Figure-1 and Figure-2.

Table-1

\begin{tabular}{|l|l|l|l|l|l|}
\hline \multicolumn{5}{|c|}{ Group Statistics } \\
\hline \multirow{2}{*}{ PSV } & Blood pressure & Number of participants & Mean & Std. Deviation & Std. Error Mean \\
\cline { 2 - 6 } & Normal & 77 & 36.6429 & 12.27306 & 1.39865 \\
\cline { 2 - 6 } & HTN & 60 & 35.6300 & 17.53961 & 2.26435 \\
\hline
\end{tabular}

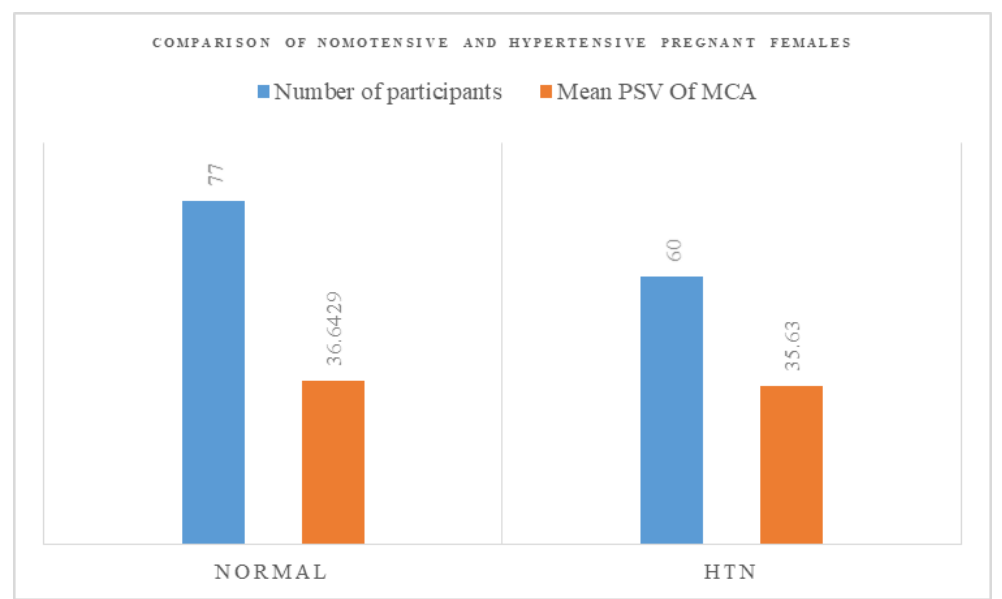

Fig-1

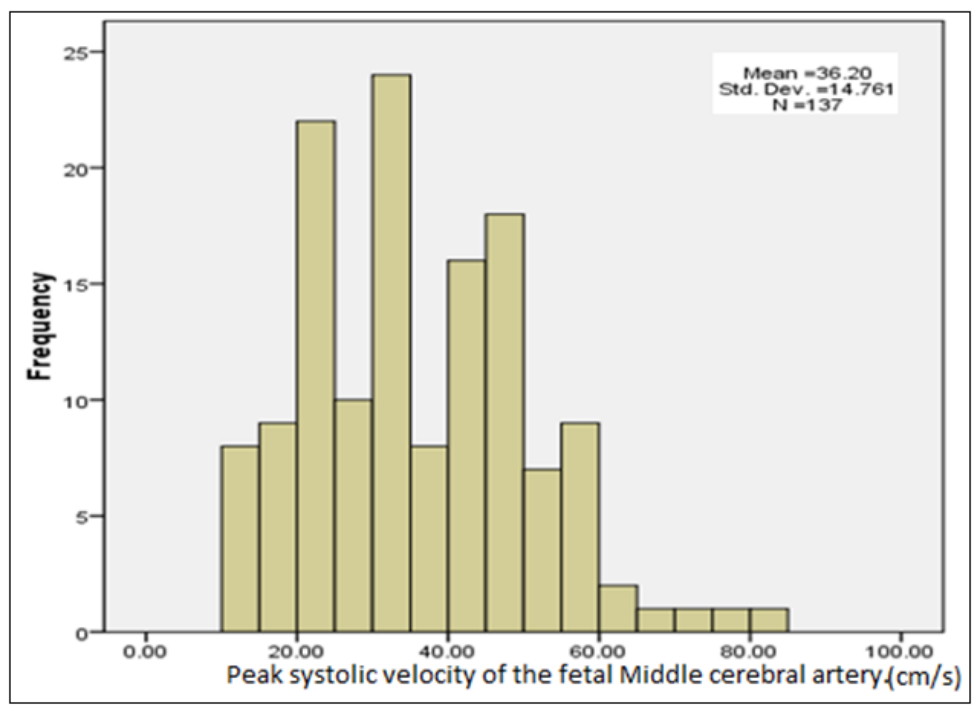

Fig-2 


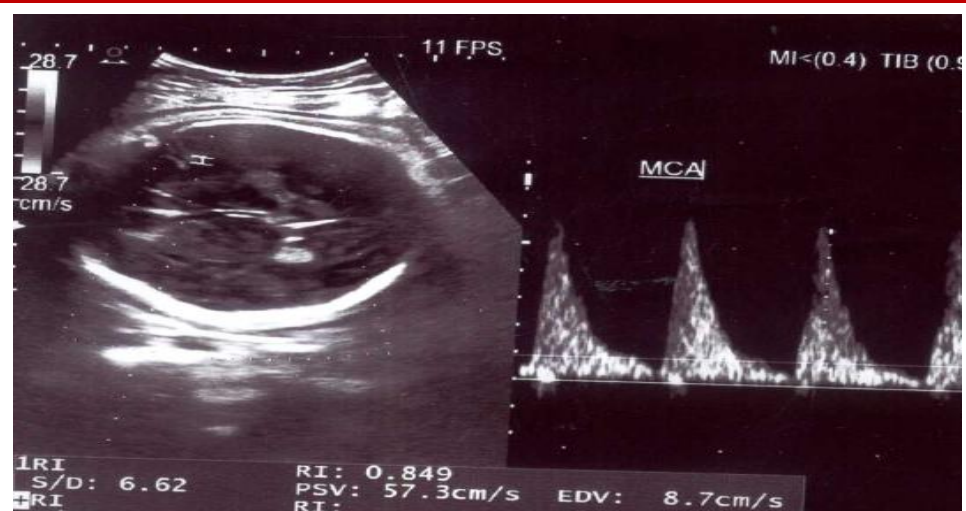

Fig-1: Peak systolic velocity of the fetal Middle cerebral Artery is $57.3 \mathrm{~cm} / \mathrm{s}$ at 30 weeks of pregnancy in normotensive mothers

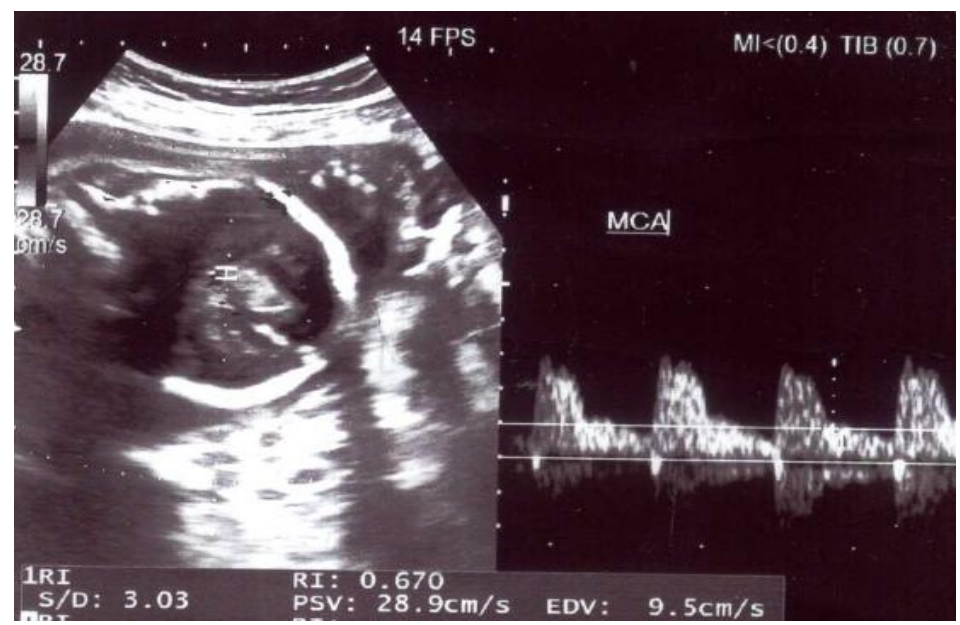

Fig-2: Peak systolic velocity of the fetal Middle cerebral Artery $28.9 \mathrm{~cm} / \mathrm{s}$ at 27 weeks of pregnancy in hypertensive mothers

\section{DISCUSSION}

This study was performed to know the relationship between increase and decrease of the peak systolic velocities of fetal middle cerebral arteries among normotensive and hypertensive mothers. Among the patients of 137, we concluded that peak systolic velocity of middle cerebral artery is reduced in hypertensive mothers.

Acute severe hypertension is caused by generalized vasoconstriction in arteries and decreased blood flow in utero-placental and resulting in insufficient supply of oxygen at places of mother's fetal exchange, forwarding the baby to lack of oxygen supply, hypoxia. The research studies submit that the assessment of blood flow in the middle cerebral artery of the fetus is one of finest signs of hazard of contrary perinatal consequence and analyzing the related diseases afterwards.

Michael Fischer, Helen and Sonia presented a research in 2015 for relating hypertension during pregnancy and abnormalities of the neonates. This cohort study involved more than eight hundred thousand pregnancies and were followed up after birth of the babies. Generally, more than 19 thousand were diagnosed with chronic hypertension; of which, $42.0 \%$ were given the treatment of hypertension by drugs.
When theses treated mothers and pregnancies were compared to the normal ones, pregnancies with hypertension treatments were at higher risks of abnormalities by birth. In their study of abnormalities of the babies' organs and body parts, the treated and untreated hypertension during pregnancy had a notable rise in the danger of heart abnormalities (OR 1.6, 95\% and OR $1.5,95 \%$ respectively). Abnormalities by birth in the neonates were seen in more than 29 thousand patients that were not hypertensive or given drugs for hypertension, four hundred and ninety one patients that were exposed to antihypertensive treatment, and five hundred and eighty one pregnancies without the treatment of pregnancy induced hypertension. Major abnormalities in the babies were of heart followed by the malformations of muscles, genitals, urinary and the digestive systems [14].

Line Sehested and Pedersen studied on the predictions and reasons for intrauterine growth retardation. 73 patients were kept under the study. $78 \%$ of the neonates were delivered by $\mathrm{C}$-section. Risk factors of mothers included hypertension during pregnancy, cigarette smoking and abruption of the placenta. 22 babies were diagnosed by hypoglycemia. After follow up of 12 months, $90 \%$ of the babies gained good health and $7 \%$ had a neurologically poor results. None of the infants were demised [15]. 
Another study was conducted by Allanson and Mari Muller in South Africa. They studied intrauterine death and related them with maternal diseases. A total of twenty three thousand, five hundred and three births were there. There were six hundred and eighty seven deaths right after delivery. The early neonatal demise was $50.7 \%$ more than deaths due to complications of mothers and then resulted in stillbirths (50.4\%) and deaths right after birth were $25.8 \%$. Mothers of babies who were died late after births were healthy. Stillbirths were noticed in the mothers who were hypertensive. Early neonatal deaths had healthy mothers. Most of the neonatal deaths occurred due to immaturity (48.7\%) plus lack of oxygen supply (40.6\%). One hundred and seventy three late deaths were due to intrauterine growth restriction and happened to have a birth weight less than the $10^{\text {th }}$ centile for gestational age [16].

\section{CONCLUSIONS}

Peak systolic velocity of fetal middle cerebral artery in hypertensive mothers is less than that of Normotensive mothers.

\section{REFERENCES}

1. Kintiraki, E., Papakatsika, S., Kotronis, G., Goulis D. G., \& Kotsis, V. (2015). Pregnancy-induced hypertension. Hormones (Athens),14(2):211-23.

2. Salmani, D., Purushothaman, S., Somashekara, S. C., Gnanagurudasan, E., Sumangaladevi, K., Harikishan, R., \& Venkateshwarareddy, M. (2014). Study of structural changes in placenta in pregnancy-induced hypertension. Journal of natural science, biology, and medicine, 5(2), 352.

3. Nelson-Piercy, C. (2015). Handbook of obstetric medicine. CRC Press.

4. Houry, D. E., \& Salhi, B. A. (2014). Acute complications of pregnancy. Rosen's Emergency Medicine: Concepts and Clinical Practice. 8th ed. Philadelphia, PA: Elsevier Saunders.

5. Palei, A. C., Spradley, F. T., Warrington, J. P., George, E. M., \& Granger, J. P. (2013). Pathophysiology of hypertension in preeclampsia: a lesson in integrative physiology. Acta physiologica, 208(3), 224-233.

6. Chaiworapongsa, T., Chaemsaithong, P., Yeo, L., \& Romero, R. (2014). Pre-eclampsia part 1: current understanding of its pathophysiology. Nature

Reviews

Nephrology, 10(8), 466.

7. Steegers, E. A., Von Dadelszen, P., Duvekot, J. J., \& Pijnenborg, R. (2010). Pre-eclampsia. The Lancet, 376(9741), 631-644.

8. Knöfler, M., \& Pollheimer, J. (2013). Human placental trophoblast invasion and differentiation: a particular focus on Wnt signaling. Frontiers in genetics, 4, 190.

9. Hasegawa, J., Nakamura, M., Hamada, S., Ichizuka, K., Matsuoka, R., Sekizawa, A., \& Okai, T. (2014). Capable of identifying risk factors for placental abruption. The journal of maternal-fetal \& neonatal medicine, 27(1), 52-56.

10. Seely, E. W., \& Ecker, J. (2014). Chronic hypertension in pregnancy. Circulation. 129(11):1254-1261.

11. Cohen, E., Baerts, W., \& van Bel, F. (2015). Brain-sparing in intrauterine growth restriction: considerations for the neonatologist. Neonatology, 108(4), 269-276.

12. Kasegaonkar, M., Patil, A. D., \& Gosavi, A. G. (2016). Comparative Study Of Fetoplacental Blood Flow Of Nor-Mal And Pregnancy Induced Hypertensive Mothers By Doppler Method, To Predict Neonatal Outcome And Mode Of Delivery. Int J Anat Res, 4(3), 2868-72.

13. 13. Berkley E, Chauhan SP, Abuhamad A, Committee SfM-FMP. Doppler assessment of the fetus with intrauterine growth restriction. American journal of obstetrics and gynecology 2012;206(4):300-8.

14. Bateman, B. T., Huybrechts, K. F., Fischer, M. A., Seely, E. W., Ecker, J. L., Oberg, A. S., ... \& Hernandez-Diaz, S. (2015). Chronic hypertension in pregnancy and the risk of congenital malformations: a cohort study. American journal of obstetrics and gynecology, 212(3), 337-e1-e14.

15. Sehested, L. T., \& Pedersen, P. (2014). Prognosis and risk factors for intrauterine growth retardation. Dan Med J, 61(4), A4826.

16. Allanson, E. R., Muller, M., \& Pattinson, R. C. (2015). Causes of perinatal mortality and associated maternal complications in a South African province: challenges in predicting poor outcomes. BMC pregnancy and childbirth, 15(1), 37. 